\title{
Thoracic Meningocele
}

National Cancer Institute

\section{Source}

National Cancer Institute. Thoracic Meningocele. NCI Thesaurus. Code C101213.

A congenital abnormality in the thoracic region of the spine in which the meninges

protrude through a defect in the spinal column. 